\title{
Le Système D'information Comptable Des PME Marocaines : Observation Des Pratiques
}

\section{Younes El Bakirdi, (Doctorant en sciences de gestion)}

Université Mohammed V - Rabat / Faculté des Sciences Juridiques,

Economiques et Sociales Souissi, Morocco

Said Radi, Professeur

Université Mohammed V - Rabat, Morocco

Doi: 10.19044/esj.2017.v13n31p222 URL:http://dx.doi.org/10.19044/esj.2017.v13n31p222

\begin{abstract}
By mobilizing the theoretical framework of the specificity of SMEs, this work tries, through the observation of accounting practices, to answer the question of the relevance (production and use) of the accounting data for the managers of Moroccan SMEs. Indeed, through semi-structured individual interviews with SME managers, accounting and financial directors of SMEs and accountants, it is about to describe the different accounting practices of SMEs on the one hand, and to highlight potential determinants that may explain the differentiation of these practices on the other hand.
\end{abstract}

Keywords : SMEs, specificity, Accounting information system, contingency

\section{Résumé}

En mobilisant le cadre théorique de la spécificité des PME, le présent travail essaie, à travers l'observation des pratiques comptables, de répondre à la question de la pertinence (production et utilisation) des données comptables pour les dirigeants des PME marocaines. En effet, à travers des entretiens individuels semi-directifs menés auprès des dirigeants de PME, des directeurs comptables et financiers de PME et des experts-comptables, il s'agit de décrire les différentes pratiques comptables des PME d'une part, et de faire ressortir des déterminants potentiels qui peuvent expliquer la différenciation de ces pratiques, d'autre part.

Mots-clés : PME, spécificité, système d'information comptable, contingence

\section{Introduction}

Il va sans dire que la place importante qu'occupent les petites et moyennes entreprises (PME) dans le tissu productif des pays n'est plus à 
démontrer (Drain, 2014 ; Chouigui, 2007). En témoigne leur prédominance numérique qui dépasse les $95 \%$ (Julien, 2008), contribuant ainsi à une création d'emploi variant entre $60 \%$ et $70 \%$ (OCDE, 2004) et constituant également une forte source d'innovation et de recherche et développement (Bergeron et al, 2010 ; D’Amboise et Bakanibona, 1990).

$\mathrm{Au}$ Maroc, la PME constitue une composante importante du tissu économique national. Elle contribue de manière positive à la création de la richesse : $95 \%$ du tissu économique national, $50 \%$ de l'investissement, $50 \%$ de l'emploi, $40 \%$ de la production, $30 \%$ des exportations et $20 \%$ de la valeur ajoutée5. Toutefois, la contribution de la PME reste largement en deçà des potentialités que cette catégorie d'entreprises peut faire valoir. Elle demeure confrontée à un ensemble de contraintes endogènes et exogènes qui freinent son évolution: fragilité des structures, faiblesses des moyens techniques et humains, complexité des procédures, croissance des exigences à l'international, difficultés d'accès au financement, etc.

Par ailleurs, il n'est pas inutile de rappeler que les outils de gestion jouent un rôle primordial dans la vie des entreprises, particulièrement les PME. L'information comptable en contexte PME mérite, à notre entendement, une attention particulière, eu égard à son importance à plus d'un titre : elle contribue à la réduction de la probabilité de faillite (Lavigne, 2002 ; Lavigne et Saint-Pierre, 2002 ; Affes et Chabchoub, 2007 ; Ghorbel, 2013), et un nombre de difficultés financières rencontrées par ce type d'entreprises est dû à une carence dans leurs systèmes d'information comptable (SIC) (Lavigne, 2002 ; Affes et Chabchoub, 2007). Ainsi, avec la mondialisation des économies et la concurrence de plus en plus sévère, les dirigeants sont amenés à substituer tout raisonnement intuitif par des outils d'analyse et de gestion formels (Lavigne, 2002 ; Lavigne et Saint-pierre, 2002).

Cependant, le thème du SIC en contexte PME suscite peu d'intérêt pour les chercheurs en sciences comptables (Ghorbel, 2013). En outre, une première lecture des recherches portant sur ce thème montre que celles-ci sont, d'une part relativement peu nombreuses, et d'autre part aboutissent à des résultats mitigés (Chapellier et Mohammed, 2010 ; Chapellier et Ben Hamadi, 2012 ; Ghorbel, 2013).

En mobilisant le cadre théorique de la spécificité des PME, le présent travail essaie, à travers l'observation des pratiques comptables, de répondre à la question de la pertinence (production et utilisation) des données comptables pour les dirigeants de PME. En effet, à travers des entretiens individuels semi-directifs menés auprès des dirigeants de PME, des

${ }^{5}$ Ces chiffres sont communiqués par l'Agence Nationale pour la Promotion de la Petite et Moyenne Entreprise (Maroc PME). 
directeurs comptables et financiers de PME et des experts-comptables, il s'agit de décrire les différentes pratiques comptables des PME d'une part, et de faire ressortir des déterminants potentiels qui peuvent expliquer la différenciation de ces pratiques, d'autre part.

\section{La PME : une organisation spécifique}

Pendant longtemps, la PME a été considérée comme une miniature des entreprises de grandes tailles, d'où le manque d'intérêt porté par les chercheurs en comptabilité à ce genre d'entreprise (Lavigne, 1996, Lavigne et Saint-Pierre, 2002, Ghorbel, 2013). Pourtant, la prolifération des recherches sur les PME a permis de constater que, au-delà de leurs petites tailles, ces dernières constituent un modèle spécifique en termes de centralisation de la gestion, de leur faible spécialisation, d'une stratégie intuitive ou peu formalisée, d'un système d'information interne simple ou peu organisé, et d'un système d'information externe simple (Chapellier et Ben Hamadi, 2012 ; Zian, 2013).

Marchesnay (1988) énumère trois traits distinctifs caractérisant les PME. Il s'agit, tout d'abord, d'une direction très personnalisée axée sur le propriétaire-dirigeant qui joue à la fois le rôle de l'entrepreneur, du manager et de l'organisateur. En second lieu, et à la différence de la grande entreprise qui agit sur son environnement, la PME subit l'influence de l'environnement dans lequel elle évolue. Enfin, les PME sont marquées par une spécialisation en termes d'activités. Dans ce même ordre d'idées, Marchesnay (1991) soutient que la spécificité des PME est incarnée, d'une part par le système de gestion, et d'autre part par les caractéristiques de l'entrepreneur.

En effet, selon Merchesnay (1991), la spécificité du système de gestion apparaît essentiellement dans quatre volets : les buts, l'organisation, les activités et l'environnement. Au sein des grandes entreprises les buts sont clairement définis et fixés d'avance. Dans les PME, par contre, les buts sont tributaires des ambitions personnelles du propriétaire-dirigeant. Sur le plan organisationnel, on note une prédominance de l'information informelle et une concentration de la décision entre les mains du propriétaire-dirigeant. La question des activités ne peut être traitée qu'en établissant une comparaison entre les grandes entreprises et les PME. Ainsi, si la grande entreprise bénéficie des économies d'apprentissage, d'échelle et de variété, la PME, quant à elle, tire sa compétitivité en se spécialisant dans des activités présentant des déséconomies d'apprentissage (innovation, créativité, etc.), des déséconomies d'échelle (secteur tertiaire) et des déséconomies de champ (micro-segmentation des marchés).

Pour mieux appréhender la spécificité de la PME, nous retenons les caractéristiques avancées par Julien (1990) à savoir : une petite taille, une centralisation de la gestion, une faible spécialisation, une stratégie intuitive 
ou peu formalisée et un système d'information interne et externe simples ou peu organisés.

De prime abord, cette question de spécificité pourrait aboutir à considérer les données comptables comme faiblement pertinentes en contexte PME. Toutefois, les recherches en la matière sont, d'une part relativement peu nombreuses et, d'autre part ne livrent pas une connaissance généralisable et sont parfois contradictoires en la matière (Chapellier et Mohammed, 2010 ; Chapellier et Ben Hamadi, 2012 ; Ghorbel, 2013). Ainsi, Une première catégorie de recherches avancent la préférence des dirigeants des PME pour les médias informels et les informations verbales, d'où un SIC rudimentaire et des outils de gestion embryonnaire dont le seul but est la production de données comptables répondant aux exigences de l'administration fiscale (Affes et Chabchoub, 2007; Chapellier, 1997; Chapellier et Mohammed, 2010; Chapellier et Ben Hamadi, 2012 ; Ghorbel, 2013). Une deuxième série de recherches confirment, par contre, qu'une majorité de PME disposent d'un système d'information pour partie comptable (Chapellier et Mohamed, 2010 ; Chapellier et Ben Hamadi, 2012).

\section{Données comptables et informations comptables}

Il sied de rappeler l'utilisation par un certain nombre de chercheurs, de manière imprécise, confuse et sans distinction rigoureuse, des expressions «donnée » et « information » (Chapellier, 1997). Si le terme «donnée » concerne la disponibilité d'une matière brute, celui d' «information » renvoie, quant à lui, à l'utilisation de cette matière dans le processus décisionnel. Dès lors, la notion d' «information» comporte deux dimensions : une « dimension objective » relative aux données disponibles et une « dimension subjective » liées à l'utilisation de ces données (Chapellier et De Montgolfier, 1995). Ce qui amène Dupuy à définir le système d'information comme "un ensemble de données pertinentes c'est-à-dire effectivement et utilement incorporées aux multiples processus décisionnels, individuels ou collectifs, conduits dans les organisations » (Dupuy, 1994, cité par Chapellier et De Montgolfier, 1995, P.1055).

En matière comptable, Chapellier et De Montgolfier (1995), en s'inscrivant dans cette même logique, insistent sur la distinction entre «donnée comptable» et «information comptable»: «Les données comptables sont des données qui, élaborées en totalité ou en partie sur la base de la comptabilité, sont disponibles dans l'entreprise et les informations comptables, les données comptables qui contribuent au processus de prise de décisions des décideurs, c'est à dire les données comptables effectivement utilisées " (Chapellier et De Montgolfier, 1995, P.1055). 
Dans ce même ordre d'idées, un SDC renvoie aux données comptables disponibles, alors qu'un SIC est composé des données comptables utilisées. Il peut être défini comme "Un ensemble organisé de structure, moyens et acteurs permettant de produire des données comptables, obligatoires et facultatives, historiques et prévisionnelles, utilisées par les dirigeants de PME pour gérer leur entreprise ». (Chapellier et Mohammed, 2010, P.3).

En adoptant cette définition nous nous inscrivons dans une approche élargie qui tient en compte aussi bien les dimensions informationnelle qu'organisationnelle du SIC. Cette approche élargie du SIC englobe trois dimensions essentielles à savoir: une dimension organisationnelle, une dimension relative à la production des données comptables, et une dimension liée à l'utilisation des données comptables.

Tableau 1 : Les dimensions du SIC

\begin{tabular}{|c|c|}
\hline Dimensions & Contenu \\
\hline $\begin{array}{l}\text { Organisation du service } \\
\text { comptable interne }\end{array}$ & $\begin{array}{c}\text { Le service comptable interne } \\
\text { Effectif du service comptable interne } \\
\text { Niveaux hiérarchiques du service comptable interne } \\
\text { Automatisation du service comptable et des activités } \\
\text { comptables }\end{array}$ \\
\hline $\begin{array}{l}\text { Production des données } \\
\text { comptables }\end{array}$ & $\begin{array}{c}\text { Pratiques de la comptabilité générale } \\
\text { Pratiques de calcul de coûts } \\
\text { Pratiques budgétaires et de gestion prévisionnelle } \\
\text { Pratiques d'élaboration de tableaux de bord } \\
\text { Pratiques d'analyse de la rentabilité } \\
\text { Pratiques d'analyse du risque } \\
\text { Pratiques de choix d'investissement et de financement }\end{array}$ \\
\hline $\begin{array}{l}\text { Diffusion des données } \\
\text { comptables }\end{array}$ & Types de destinataires \\
\hline $\begin{array}{l}\text { Utilisation des données } \\
\text { comptables }\end{array}$ & $\begin{array}{c}\text { Données comptables utilisées } \\
\text { Objectifs d'utilisation }\end{array}$ \\
\hline
\end{tabular}

Source : Etabli par nos propres soins ${ }^{6}$

Par ailleurs, la production et l'utilisation de l'information sont des actes de nature organisationnelle. Elles dépendent des contextes structurel et comportemental dans lesquels évolue l'organisation. La théorie de la contingence est mobilisée par les chercheurs pour expliquer la différenciation du SIC des PME (degrés de complexité inégaux). Des facteurs de contingence peuvent influencer le système d'information comptable (Affes et Chabchoub, 2007). Eu égard aux spécificités de la PME, la théorie de la contingence sous-entend l'influence de facteurs structurels et comportementaux sur l'existence de configurations diverses du système

${ }^{6}$ Etabli pas nos propres soins sur la base des travaux de Chapellier (1994), Mohammed (2010) et Chapellier et Ben Hamadi (2012). 
d'information comptable (Chapellier et De Montgolfier, 1995; Affes et Chabchoub, 2007 ; Chapellier et Mohammed, 2010 ; Ghorbel, 2013). Ceci étant, bien que l'ensemble des études confirment l'influence d'un certain nombre de facteurs sur la différenciation des SIC de PME, il n'en demeure pas moins l'absence de consensus autour de ces déterminants.

\section{Méthodologie}

Notre objectif à travers cette étude est d'explorer un domaine jusquelà peu étudié au Maroc à savoir le SIC des PME marocaines. Nous jugeons à cet égard que l'enquête qualitative est un moyen adéquat pour répondre à cet objectif (Gavard-Perret et al, 2012).

Parmi les techniques de collecte de données qualitatives les plus utilisées nous avons opté pour l'entretien individuel semi-directif qui paraît, à notre sens, le mieux adapté à notre objectif d'explorer le SIC des PME marocaines et de mettre en évidence la différenciation des pratiques. Ce choix est justifié par au moins trois raisons. Tout d'abord, il s'agit de dévoiler l'objet de recherche afin de pouvoir collecter des données plus centrées. Ensuite, nous avons affaire à une relation individuelle avec les personnes interrogées. Enfin, cette étude a pour objectif de collecter des données de "signe» qui "font référence à des données collectées directement, par l'expression verbale » (Gavard-Perret et al, 2012, P.108).

Ainsi, à l'aide d'un guide d'entretien, dix entretiens individuels semidirectifs ont été menés auprès de deux catégories d'acteurs :

La première catégorie d'acteurs est composée des témoins privilégiés : "Il s'agit de personnes qui, par leur position, leur action ou leurs responsabilités, ont une bonne connaissance $d u$ problème.» (Campenhoudt et Quivy, 2011, p. 60). En effet, des entretiens sont menés auprès de quatre experts-comptables qui constituent des interlocuteurs dont l'activité professionnelle les amène à avoir une bonne connaissance du SIC des PME ;

- $\quad$ La deuxième catégorie d'acteurs est constituée du public directement concerné par l'étude à savoir les dirigeants et les comptables internes des PME. A cet égard, six entretiens ont été menés auprès de quatre dirigeants et de deux responsables de services comptables et financiers des PME.

S'agissant de la définition de la PME, eu égard à leur grande diversité, il est toujours difficile de construire des typologies opérationnelles de PME. Toutefois, les chercheurs se sont efforcés de trouver des ressemblances en se basant sur un certain nombre de critères tant quantitatifs que qualitatifs (Julien, 1990). Au Maroc, la charte de la PME de 2002 établit une distinction entre les entreprises existantes et les entreprises nouvellement créées. 
Tableau 2 : Définition selon la charte de la PME

\begin{tabular}{|c|c|c|c|c|}
\hline \multicolumn{3}{|c|}{ Entreprises existantes } & \multicolumn{2}{|c|}{ Entreprises nouvellement créées* } \\
\hline $\begin{array}{c}\text { Effectif } \\
\text { permanent }\end{array}$ & $\begin{array}{c}\text { Chiffre } \\
\text { d'affaires } \\
\text { annuel HT** }\end{array}$ & $\begin{array}{l}\text { Ou otal bilan } \\
\text { annuel } * * *\end{array}$ & $\begin{array}{c}\text { Programme } \\
\text { d'investissement } \\
\text { initial }\end{array}$ & $\begin{array}{l}\text { Ratio d'investissement } \\
\text { par emploi }\end{array}$ \\
\hline$\leq 200$ & $\begin{array}{c}\leq 75 \text { millions } \\
\text { de DH }\end{array}$ & $\begin{array}{c}\leq 50 \text { millions } \\
\text { de } \mathrm{DH}\end{array}$ & $\begin{array}{c}\leq 25 \text { millions de } \\
\text { DH }\end{array}$ & $\leq 250.000 \mathrm{DH}$ \\
\hline
\end{tabular}

Source : Etabli par nos propres soins

*Toute entreprise ayant moins de deux années d'existence

**Au cours des deux derniers exercices

*** Au cours des deux derniers exercices

Actuellement, le contrat-programme (2015-2020) entre l'Etat et Maroc PME, retient le chiffre d'affaires comme seul critère pour catégoriser les entreprises pouvant bénéficier des programmes d'appui et d'accompagnement. En effet, selon ce nouveau contrat-programme, est considérée PME toute entreprise réalisant un chiffre d'affaires annuel hors taxe entre 10 et 200 millions de dirhams.

Nous avons retenu le critère « effectif employé » pour définir la PME malgré le fait qu'il pose un certain nombre de limites inhérentes à la fois au sens que l'on peut attribuer à cette notion (employés permanents, saisonniers, etc.) ainsi qu'aux différences sectorielles constatées (Julien, 1990). En effet, au sens de cette étude, une PME est toute entreprise employant un effectif compris entre 10 et 200 salariés.

Tableau 3 : Les caractéristiques des entreprises étudiées

\begin{tabular}{|c|c|c|c|c|c|}
\cline { 2 - 6 } \multicolumn{1}{c|}{} & $\begin{array}{c}\text { Année de } \\
\text { création }\end{array}$ & $\begin{array}{c}\text { Statut } \\
\text { juridique }\end{array}$ & $\begin{array}{c}\text { Effectif } \\
\text { employé }\end{array}$ & Activité $^{7}$ & $\begin{array}{c}\text { Personne } \\
\text { interviewée }\end{array}$ \\
\hline PME 1 & 2007 & SARL & 15 & $\begin{array}{c}\text { Programmation et } \\
\text { conseil informatiques }\end{array}$ & Dirigeant \\
\hline PME 2 & 2005 & SARL & 40 & Commerce de gros & Dirigeant \\
\hline PME 3 & 2014 & SARL.AU & 88 & $\begin{array}{c}\text { Programmation et } \\
\text { conseil informatiques }\end{array}$ & $\begin{array}{c}\text { Responsable } \\
\text { financier et } \\
\text { comptable }\end{array}$ \\
\hline PME 4 & 1984 & SA $^{10}$ & 94 & $\begin{array}{c}\text { Fabrication de } \\
\text { margarine }\end{array}$ & Dirigeant \\
\hline PME 5 & 1983 & SA & 200 & $\begin{array}{c}\text { Edition de logiciels / } \\
\text { Gestion d'installations } \\
\text { informatiques }\end{array}$ & $\begin{array}{c}\text { Responsable } \\
\text { financier et } \\
\text { comptable }\end{array}$ \\
\hline PME 6 & 1990 & SA & 200 & Transport & Dirigeant \\
\hline
\end{tabular}

Source : Etabli par nos propres soins.

7 La détermination des activités est basée sur la nomenclature marocaine des activités économiques (NMA 2010) approuvée par le décret $n^{\circ}$ 2-12-481 du 14 hija 1433 (30 octobre 2012).

${ }^{8}$ Société à Responsabilité Limitée.

${ }^{9}$ Société à Responsabilité Limitée à Associé Unique.

${ }^{10}$ Société Anonyme. 


\section{Résultats et discussion}

Les renseignements des entretiens avec les témoins privilégiés et le public-cible : une différenciation du SIC des PME

\section{Organisation du service comptable interne}

Il ressort des entretiens menés avec les experts-comptables que l'infrastructure comptable des PME est différenciée. En effet, en termes d'existence de service comptable interne, on assiste à l'existence de deux catégories de PME. D'une part, une première catégorie qui dispose d'un service comptable interne et, d'autre part, une deuxième catégorie, sans service comptable interne, et externalisant la totalité de sa comptabilité auprès des comptables externes.

Cette différenciation est présente également au sein des PME disposant d'un service comptable interne. Une différentiation qui porte aussi bien sur l'effectif que sur les niveaux hiérarchiques. Ainsi, certaines PME n'ont qu'une seule personne chargée de la comptabilité, tandis que d'autres disposent d'une direction financière et comptable avec plusieurs niveaux hiérarchiques et une distinction entre la comptabilité et la gestion. Dans ce sens, un expert-comptable affirme ce qui suit :

"En ce qui concerne le service comptable il y'a généralement deux cas: soit un service comptable avec une seule personne soit une vraie direction financière avec un chef comptable, des comptables confirmés et un contrôleur de gestion».

S'agissant de l'automatisation du service et des activités comptables, les PME qui tiennent leur comptabilité en interne sont outillées d'un logiciel comptable. Toutefois, les PME ne sont pas toutes dotées d'un logiciel comptable intégré. Les entretiens avec les experts-comptables montrent que, généralement, la comptabilité en PME est manuelle dans le système comptable. Manuelle dans le sens où les services «métier» passent des opérations et transmettent par la suite les pièces comptables au service comptable interne qui les comptabilise dans le logiciel comptable. Toutefois, on assiste de plus en plus à une gestion intégrée de la comptabilité (à travers les $\mathrm{ERP}^{11}$ ). Autrement dit le métier saisit les opérations dans une application qui lui est propre, il valide et automatiquement la comptabilité est générée sans passer manuellement les écritures comptables.

Les entretiens menés avec les dirigeants et les responsables financiers et comptables ont permis de soulever une hétérogénéité en matière d'organisation du service comptable interne. En effet, La PME 1 ne dispose pas d'un service comptable interne et externalise toute sa comptabilité auprès d'un comptable externe. La PME 2 et la PME 3 mettent en place un service comptable de deux à trois personnes. La PME 4, la PME 5 et la PME 6

\footnotetext{
${ }^{11}$ ERP : Enterprise Resource Planning (progiciel de gestion intégré).
} 
disposent, quant à elles, d'une vraie direction financière et comptable avec plusieurs niveaux hiérarchiques et une distinction comptabilité / gestion.

Mis à part la PME 1 où presque toutes les activités comptables sont menées par le comptable externe, ce sont les services comptables et financiers internes qui s'en occupent pour les autres PME. Néanmoins, les PME 4, 5 et 6 établissent une séparation en termes de personnes en charge. Ainsi, le chef comptable et les aides comptables s'occupent des activités telles que la journalisation des opérations, la tenue des comptes, la préparation des états financiers annuels et intermédiaires, la comptabilité fournisseurs et clients, la trésorerie et la fiscalité. Par ailleurs, le contrôleur de gestion est chargé du contrôle des stocks, de la gestion budgétaire, et de la préparation des reporting mensuels.

Le principal acteur fortement présent en matière de prise de décisions liées au SIC est le dirigeant, notamment en ce qui concerne les PME 1 et 2. A cet égard, il choisit le responsable et les employés du service comptable, il décide du recours à l'expert-comptable et il décide de l'informatisation de la comptabilité. Par ailleurs, le responsable comptable interne est beaucoup plus présent dans les autres PME. A titre d'exemple, les responsables des services comptables et financiers des PME 3, 4 et 5 sont en charge du choix des employés du service comptable, de la décision de recourir au comptable externe, de la proposition du cabinet d'expertise-comptable, de la définition des postes et des tâches et de l'informatisation de la fonction comptable.

S'agissant de l'automatisation de la comptabilité, toutes les PME disposent d'un logiciel de comptabilité à l'exception de la PME 1. En matière de disponibilité d'un logiciel comptable intégré, à part la PME 2, les autres PME ont en disposent, ce qui permet l'informatisation des principales applications comptables telles que la tenue des journaux et du grand livre, les états de synthèse, la gestion de la trésorerie, la facturation et la gestion du stock.

Selon les personnes interviewées, plusieurs facteurs sont à l'origine de cette différenciation en termes d'infrastructures comptables mises en place. La taille de l'entreprise est, sans conteste, le premier élément soulevé. C'est ce qui ressort des propos de la personne responsable du service comptable de la PME 3 :

"Au début, il n'y avait que dix salariés. Nous n'avions pas besoin de mettre en place un service comptable. Avec l'augmentation du volume de notre activité, on a mis en place un service qui s'occupe à la fois des affaires administratives et financières et qui est dirigé par une seule personne. En raison de la croissance de notre activité, nous avons renforcé ce service par le recrutement d'une deuxième personne qui aide le 
responsable. Nous allons embaucher très prochainement une autre personne pour s'occuper des affaires administratives ».

En effet, Plus l'entreprise est grande plus on assiste à l'existence d'un service comptable interne. Selon la taille, il y a trois catégories d'entreprises: i) celles qui externalisent la comptabilité et la paie directement à l'expert-comptable, ii) celles qui tiennent uniquement la comptabilité plus les déclarations fiscales en interne, et qui externalisent la paie, et iii) celles qui internalisent tout chez elles et chargent l'expertcomptable de la supervision et le contrôle afin de se rassurer en matière de la tenue de la comptabilité, la fiscalité et le social.

Ensuite, le profil du dirigeant est avancé comme étant un facteur susceptible d'influencer la différentiation de l'infrastructure comptable. D'après un expert-comptable, si le dirigeant n'a pas de connaissances en comptabilité et fiscalité, il est incapable de vérifier la fiabilité des données qui lui sont transmises par le comptable interne. Plusieurs PME qui disposent d'un service comptable interne ont des dirigeants formés en comptabilité et gestion. Le profil du comptable interne est également un élément qui peut expliquer cette différenciation puisque même si le dirigeant recrute un comptable interne il n'est pas sûr de son degré de compétence. Un expertcomptable nous a informés sur le cas d'une PME qui a eu un redressement fiscal dû à un certain nombre d'erreurs comptables commises par son comptable interne.

\section{Production et diffusion des données comptables}

A l'instar des infrastructures comptables, les entretiens avec les témoins privilégiés et le public-cible montrent que la différentiation porte aussi sur la production des données comptables et concerne l'ensemble des pratiques.

\section{Pratiques de la comptabilité générale}

En matière de pratiques de comptabilité générale, les expertscomptables interviewés avancent que, bien que toutes les PME préparent leurs états financiers annuels puisqu'il s'agit d'une obligation légale, rares sont celles qui établissent des états financiers intermédiaires. C'est ce qui ressort également des entretiens avec le public-cible. Ainsi, si les PME 1 et 2 n'ont jamais élaboré d'états financiers intermédiaires, les PME 3 et 6 les préparent parfois, tandis que les PME 4 et 5, en plus des états intermédiaires semestriels obligatoires, produisent des états financiers intermédiaires mensuels.

Plusieurs facteurs peuvent expliquer cette différenciation. Il s'agit en premier lieu de la taille de l'entreprise. Selon un expert-comptable, ces arrêtés intermédiaires mensuels, trimestriels ou semestriels s'imposent pour 
les PME de grande taille sinon elles ne peuvent pas suivre le volume important des activités et des effectifs.

L'endettement de l'entreprise peut aussi expliquer l'élaboration des états financiers intermédiaires dans la mesure où, pour contracter un crédit, certains dirigeants de PME, à la demande de leurs banquiers, préparent un business plan et des états financiers intermédiaires et prévisionnels. A cet égard, le dirigeant de la PME 6 précise que :

«La production des états financiers intermédiaires n'est pas une pratique courante au sein de notre entreprise, mais ça nous arrive de préparer des états financiers intermédiaires à la demande de la banque pour le renouvellement de notre ligne de crédit $\gg$.

Il ressort des entretiens que le statut juridique de l'entreprise est un facteur qui peut expliquer cette différenciation dans la mesure où les PME 4, 5 et 6 sont tenus juridiquement de produire des états financiers semestriels du fait qu'elles sont des sociétés anonymes. En outre, la PME 5 élabore des états financiers mensuels avec un bilan et un compte de résultat mensuels et le calcul de l'IS mensuel.

Le profil du dirigeant peut jouer un rôle dans le recours à ce genre de pratiques. Selon un expert-comptable, les dirigeants jeunes ou ayant bénéficié d'une formation en comptabilité /gestion sont très impliqués dans la gestion et ont le souci du suivi et du contrôle. Pour cela, ils établissent des arrêtés trimestriels voir mensuels pour faire l'état des lieux d'un certain nombre d'indicateurs tels que le chiffre d'affaires et le résultat et prendre les décisions qui s'imposent.

\section{Pratiques de la comptabilité analytique}

Les experts comptables mettent en exergue deux catégories de dirigeants en matière de pratiques de calcul de coûts. Il y a, d'une part, des dirigeants qui, faute de mise en place d'un système formalisé de calcul de coûts, s'appuient sur leur intuition pour calculer les marges et fixer les prix de vente. C'est un calcul qui n'est pas nécessairement juste car il n'inclut pas toutes les composantes des coûts. D'autre part, il existe d'autres dirigeants, non majoritaires, qui établissent un vrai système de comptabilité analytique basé sur un calcul rigoureux et détaillé des différents coûts. Plusieurs méthodes sont adoptées tels que le coût complet ou le coût partiel, mais c'est la méthode des coûts complets qui est généralement pratiquée.

Différentes pratiques de calcul de coûts ont été remarquées au sein des PME concernées par l'étude. Pour les PME 1 et 3 qui sont des entreprises de services, la masse salariale constitue la principale composante pour le calcul de coûts. Pour la PME 1, le calcul de coûts est basé sur «le taux moyen journalier (TMJ) » de rémunération par profil employé, tout en 
lui ajoutant les coûts communs liés aux ressources supports, aux achats et aux frais divers. Pour la PME 3, le calcul des coûts se fait par projet sachant que le principal coût est celui des salariés. En effet, le responsable comptable interne dresse mensuellement un tableau contenant le coût complet par salarié et par projet (salaire brut, net, primes) et tout ce qui est en commun le répartit sur les différents projets (loyer, eau et électricité, connexion internet, etc.).

La PME 2, spécialisée dans le commerce de gros des articles importés, adopte la méthode des coûts partiels. En effet, le coût est calculé par produit, à chaque nouvel arrivage et d'une manière très détaillée. Ainsi, le coût de chaque produit est composé de son prix d'achat (tenant en considération le cours de change) augmenté des frais de transport (jusqu'au port et du port jusqu'aux dépôts de stockage) et des charges variables (transitaire,...).

Les PME 4 et 5 quant à elles, établissent un système de calcul de coûts complets avec la mise en place d'une méthode d'évaluation du stock et procèdent également au calcul du seuil de rentabilité. A titre d'exemple, pour la PME 5, il y a une répartition analytique dans le logiciel comptable à travers la mise en place des centres de coûts par activité avec les produits y afférents. Les charges indirectes sont calculées suivant une répartition selon leurs quotte part par activité y compris les dotations d'amortissement et les frais financiers. En ce qui concerne la gestion des stocks, c'est la méthode du coût moyen unitaire pondéré qui est adoptée.

Il ressort de ce qui précède une différenciation en termes de pratiques relatives à la comptabilité analytique. Une différentiation qui semble être liée, tout d'abord, à la taille de l'entreprise dans la mesure où les PME ayant un système de calcul de coûts élaboré sont des entreprises de grandes tailles et dotées d'une direction comptable et financière avec la présence d'un service de contrôle de gestion. Ensuite, et eu égard aux constats dressés plus haut, les pratiques de calcul différent selon que la PME exerce dans le secteur de services (PME 1 et 3) ou industriel (PME 4 et 5).

\section{Pratiques de contrôle de gestion}

Les experts-comptables constatent que les pratiques budgétaires, de gestion prévisionnelle et de tableaux de bord sont très rarement produites dans les PME. Les entretiens avec le public-cible montrent une différenciation desdites pratiques. En effet, les pratiques budgétaires et de gestion prévisionnelle sont absentes dans les PME 2 et 6 . Les PME 1 et 3 établissent essentiellement des budgets de trésorerie. Par contre, les PME 4 et 5 préparent des budgets prévisionnels annuels (répartis mensuellement) très élaborés et couvrant l'ensemble des activités (Achat, production, vente, distribution, investissement, projet, Trésorerie) avec l'élaboration d'un bilan 
et d'un compte de résultat prévisionnels. Un suivi des prévisions budgétaires est assuré également par le service de contrôle de gestion qui élabore un reporting mensuel dressant une analyse comparative entre les réalisations et les prévisions (analyse des écarts). S'agissant des pratiques de tableaux de bord, les PME 1 et 3 ne tiennent pas des tableaux de bord. Les autres PME disposent toutes de tableaux de bord opérationnels dont le contenu différent d'une entreprise à une autre. Par ailleurs les PME 4 et 5 s'inscrivent dans un projet de mise en place d'un tableau de bord prospectif.

La taille de l'entreprise semble être déterminante de cette différenciation puisque du fait que, si le volume d'activité est maitrisable, il n'y a pas un besoin réel d'instaurer un système de comptabilité budgétaire. Par contre, une fois les flux de volume deviennent très importants, il est nécessaire de créer une unité à part qui assure le suivi de ces indicateurs. C'est le cas des PME 4 et 5 qui disposent d'une direction des affaires comptables et financières avec une distinction entre les fonctions comptabilité et gestion avec la présence d'un service formel dédié au contrôle de gestion.

\section{Pratiques d'analyse financière et du choix d'investissement et de financement}

Ces pratiques sont très rarement présentes au sein des PME selon les experts- comptables interviewés. C'est ce qui ressort également des entretiens avec le public cible puisqu'elles ne sont présentes que dans les PME 4 et 5. Il s'ensuit qu'en matière d'analyse de la rentabilité ces deux entreprises élaborent un reporting mensuel contenant les soldes intermédiaires de gestion, la capacité d'autofinancement, un certain nombre de ratios (poids des achats par rapport au chiffre d'affaires, rentabilité de l'activité, la rentabilité du personnel, la rentabilité du matériel, le poids des charges financières). Une analyse comparative de la rentabilité dans le temps et dans l'espace est également présente. A cet égard, le dirigeant de la PME 4 établit une analyse de la rentabilité aussi bien par rapport aux années passées (2 à 3 ans), que par rapport aux concurrents en éditant leurs états financiers.

S'agissant des pratiques d'analyse du risque, on constate la présence dans ces deux PME (4 et 5), avec une fréquence mensuelle, des tableaux de financement, du calcul du besoin en fonds de roulement normatif et de plusieurs ratios tels que la solvabilité, l'autonomie financière, le délai de rotation des stocks, le délai de rotation des créances clients et le délai de rotation des dettes fournisseurs. De la même manière, les pratiques de choix d'investissement et de financement ne sont présentes que dans ces deux PME où il $\mathrm{y}$ a des calculs systématiques de la valeur actuelle nette (VAN), du taux interne de rentabilité (TIR) et du délai de récupération. 
Plusieurs facteurs peuvent être avancés pour expliquer cette différentiation de pratiques. Il s'agit, en premier lieu de l'endettement dans la mesure où ces pratiques sont élaborées en cas de demande de crédit bancaire. c'est le cas pour la PME 1 qui a élaboré des indicateurs d'analyse de la rentabilité, du risque et du choix d'investissement et de financement une seule fois au moment de la présentation de sa candidature pour bénéficier d'un financement (crédit) dans le cadre des programmes d'accompagnement mis en place par Maroc PME. Dans ce même ordre d'idées, Ces données ont été demandées à la PME 3 par sa banque pour sa demande de crédit ».

Le deuxième élément qui peut influencer ces pratiques est le profil du dirigeant. Ainsi, une différence est constatée au niveau du type et niveau de formation des dirigeants. En effet, les dirigeants des PME 1 et 3 sont des ingénieurs, celui de la PME 2 est un bachelier alors que le dirigeant de la PME 6 a effectué des études supérieures en droit. Par contre, le dirigeant de la PME 4 (où il y a existence de ces pratiques) est titulaire d'un diplôme d'études supérieures en audit et contrôle de gestion.

Le troisième facteur d'impact peut être inhérent au profil de la personne responsable du service comptable interne dans la mesure où les PME 4 et 5 caractérisées par la présence systématique de ces pratiques disposent toutes les deux d'une direction financière et comptable dirigée par une personne ayant fait des études supérieurs en comptabilité et gestion et des collaborateurs avec des qualifications élevées en la matière.

\section{Diffusion des données comptables}

La différenciation des pratiques concerne également la diffusion des données comptables. Pour certaines PME, la communication interne des chiffres est confidentielle est plutôt l'affaire du dirigeant, du comptable interne et du comptable externe. Pour d'autres, par contre, les autres services accèdent aux données comptables. Selon un expert-comptable, le cloisonnement ou l'ouverture en termes de diffusion des données comptables n'émanent pas toujours du dirigeant, ils émanent aussi du comptable interne qui veut garder son «patrimoine » comme frontières infranchissables. De ce fait, il ne veut pas partager l'information, parfois il trouve que cela va le rendre indispensable.

La diffusion en interne des données comptables n'est pas une pratique très répandue au sein des PME 2 et 3 . Les trois principaux destinataires des données comptables sont le dirigeant, le comptable interne et le comptable externe. Ainsi, il n'y a pas de variété de destinataires des données comptables au sein de la PME 2. En fait, chaque service assure la production des données qui les concerne sans toutefois que les autres services aient un droit de regard sur le contenu. Les données ainsi produites sont centralisés dans le logiciel comptable et le dirigeant est le seul qui en a 
l'accès. La PME 3 s'inscrit dans la même logique. Son responsable du service comptable énonce les propos suivants :

"Les deux principaux destinataires sont le dirigeant et l'expert-comptable. Il s'agit de données secrètes de la société qu'aucune personne ne doit en avoir connaissance. En ce qui concerne la fréquence de diffusion, deux cas se présentent. En ce qui concerne le dirigeant, un rapport mensuel lui est soumis. S'agissant de l'expert-comptable, les données comptables lui sont communiquées à la fin de l'année en vue d'établir les états annuels ».

Toutefois, une exception est constatée au sein de la PME 1 que son dirigeant ne voit pas de problème en termes de diffusion des données comptables auprès du personnel de l'entreprise. Par contre, il existe une communication auprès de l'ensemble de l'équipe employée sur les rubriques consommatrices et les projets rentables.

\section{Utilisation des données comptables}

En matière d'importance accordée aux données comptables, tous les experts-comptables interrogés ont souligné l'existence de deux catégories de dirigeants de PME. La première catégorie est constituée de dirigeants qui ne considèrent la comptabilité que comme servant pour la préparation des états financiers pour les déclarations fiscales. Une deuxième catégorie, par contre, se compose de dirigeants qui accordent de l'importance aux données comptables pour la gestion et le pilotage de leurs entreprises.

Pour la première catégorie de dirigeants, la comptabilité n'est pas considérée comme un outil de gestion, de pilotage et d'appréciation des performances. En effet, pour eux le bilan ne reflète pas la réalité soit parce qu'ils exercent des activités qui ne sont pas forcément déclarées avec les vrais chiffres, ou bien gonflent les charges pour des raisons d'évasion fiscale. Un expert-comptable précise à cet égard :

"L'année dernière, j'ai eu l'occasion de tenir la comptabilité

d'une PME. Après six mois, j'ai établi un reporting semestriel que j'ai adressé au dirigeant. Ce dernier m'a informé que cela fait vingt ans qu'il dirige son entreprise sans jamais réaliser un reporting de gestion $»$.

Toutefois, cette vision commence à changer selon les expertscomptables. On assiste aujourd'hui à une deuxième catégorie de dirigeants de PME qui utilisent les données comptables pour le pilotage et la gestion de leurs entreprises, en calculant les taux de marge, l'évolution des différents produits et charges, et en analysant les causes de la baisse ou de l'augmentation des résultats sur la base des reporting mensuels. 
Encore une fois une différenciation a été constatée en termes des rôles assignés au dispositif comptable, à l'utilisation des données comptables et aux objectifs d'utilisation. En effet, bien que l'ensemble des PME étudiées utilisent les données comptables à des degrés différents et à des objectifs différents, il n'en demeure pas moins que le souci fiscal ressort comme premier motif derrière la production des données comptables. C'est ce qui émane par exemple des propos du dirigeant de la PME 2 :

"A cause d'un certain nombre d'incidents du passé (erreurs commises par le comptable externe), le service comptable interne, en menant en parallèle le même travail réalisé par le comptable externe, procède au contrôle et établit une comparaison avec les livrables de celui-ci. Par la suite ce sont nous qui faisons les déclarations auprès du fisc. Nous avons en interne les compétences nécessaires pour faire ce travail sans recours au comptable externe, mais la mise en place de cette méthode de travail me permet de verrouiller le système en mettant en place deux circuits en parallèles afin de limiter les risques au maximum parce que nous avons affaire à l'administration fiscale».

Par rapport à l'utilisation des données comptables, à l'exception des PME 4 et 5 qui ont une production diversifiées et contenant l'ensemble des composantes du système de données comptables, toutes les autres entreprises se limitent aux données incluses dans le bilan, le compte de résultat et les rapports de coûts. Pour le dirigeant de la PME 2, le dispositif comptable joue un rôle très important dans la mesure où il l'aide à faire ressortir de l'information relative à un certain nombre d'indicateurs tels que le chiffre d'affaires mensuel par commercial, le chiffre d'affaires annuel, les produits qui se commercialisent facilement ainsi que les produits en mal de vente, la classification des familles de produits selon leur importance, la connaissance de la marge bénéficiaire par produit, etc. Les principales données comptables utilisées par le dirigeant de la PME 3 sont celles issues du compte résultat, celles contenues dans les rapports de coûts, ainsi que les informations qui figurent dans les prévisions budgétaires. Ces données lui servent beaucoup dans les décisions de recrutement, la connaissance de la situation de la trésorerie et la constatation des budgets prévus pour les mois à venir ». Un troisième niveau d'utilisation à la fois fréquente et diversifiée est remarqué au sein des PME 4 et 5 où les données relatives à la comptabilité générale, à la comptabilité analytique, au contrôle de gestion, à l'analyse financière et aux choix d'investissement et de financement sont toutes utilisées d'une manière systématique. A titre d'exemple les reporting mensuels préparés par la direction financière et comptable permettent au dirigeant de la PME 4 d'atteindre plusieurs objectifs. Il s'agit par exemple de 
l'établissement des prévisions du CA, de la trésorerie et du résultat, de la connaissance des dus fournisseurs et clients, du choix de maintenir ou de renouveler une machine, du choix d'investir dans une activité nouvelle, du choix d'un mode de financement, de la connaissance de l'évolution des performances de l'entreprise et de la surveillance de l'évolution de la performance des concurrents, etc.

Plusieurs facteurs peuvent expliquer cette différence en matière de recours aux données comptables. Il s'agit en premier lieu de la taille de l'entreprise. Autrement dit, plus la taille de la PME est importante plus les dirigeants ont tendance à se séparer de la dualité fiscalité /comptabilité et gestion et plus ils ont tendance à utiliser les données comptables à des fins de gestion. Dans ce même ordre d'idées, les PME qui tiennent leur comptabilité en interne utilisent les données comptables à des fins de gestion alors que celles qui externalisent la totalité de leur comptabilité (PME de petite taille) considèrent cette dernière comme uniquement une obligation fiscale.

Le secteur d'activité est de nature à influer sur l'utilisation des données comptables dans la mesure où il existe des secteurs comme le commerce où les états financiers établis ne reflètent pas forcément la réalité des actions menées puisqu'on ne déclare pas la totalité des activités. Un expert-comptable utilise les propos suivants pour décrire cette situation :

"Il existe des secteurs où on peut constater une concomitance et une conformité entre la comptabilité et la gestion. Par contre dans d'autres secteurs, notamment ceux caractérisés par un degré élevé d'informel, un cloisonnement est constaté entre le volet comptable et le volet fiscal. Généralement on établit un bilan fiscal, un bilan comptable et un autre bilan bancaire. "

L'environnement de l'entreprise est également souligné comme facteur pouvant expliquer la différenciation en matière de pertinence des données comptables. Les entreprises qui sont confrontées à une réglementation et à un marché compétitif soit localement soit à l'international sont amenées à améliorer leurs systèmes d'information par l'introduction des nouvelles technologies et par l'adoption d'un système comptable intégré et correspondant à la réalité.

Le profil du dirigeant peut être un facteur explicatif de cette différence. Un expert-comptable précise dans ce sens que les entrepreneurs jeunes ont plus tendance à utiliser les outils de gestion et manifestent un intérêt particulier aux données comptables pour le pilotage de leurs entreprises (élaboration des business plan et des reporting de gestion). Le type de formation peut également être déterminant dans la mesure où, selon un autre expert-comptable, les dirigeants qui ont fait des études en comptabilité ou en gestion demandent et utilisent des données comptables sophistiquées tels que les ratios, l'analyse financière, etc. 


\section{Discussion}

\section{Pertinence des données comptables pour les dirigeants des PME}

Ce papier s'inscrit dans la problématique de la pertinence des données comptables pour les dirigeants des PME. Autrement dit, il s'agit d'apporter des éléments de réponse à la question suivante : le système d'information des dirigeants des PME marocaines est-t-il comptable ?

L'observation des dimensions retenues pour caractériser le SIC permet d'aboutir aux constats suivants :

En matière d'infrastructures comptables, la majorité des PME observées disposent de services comptables internes. Toutefois, ces derniers ont généralement un effectif réduit et une faible spécialisation, sont automatisés et pilotés par des personnes chargées de l'ensemble des activités comptables. Le dirigeant demeure l'acteur comptable central dans la mesure où il est concerné par la majorité des décisions comptables ;

- $\quad$ S'agissant de la production des données comptables, l'étude montre que les PME ne se limitent pas uniquement à la production des données comptables obligatoires pour se conformer aux dispositions fiscales. On assiste à l'élaboration des données comptables de gestion telles que celles inhérentes aux calculs de coûts, aux budgets et gestion prévisionnelle et aux tableaux de bord opérationnels. Cependant, une faiblesse est constatée en matière de pratiques d'analyse financière et de choix d'investissement et de financement.

Les dirigeants des PME étudiées sont conscients de l'importance des données comptables. A cet effet, les données comptables semblent être pertinentes pour les dirigeants des PME dans la mesure où elles sont effectivement utilisées par eux pour piloter leurs entreprises.

A l'instar d'autres recherches (Chapellier, 1994; Affes et Chabchoub, 2007 ; Mohammed, 2010 ; Chapellier et Benhammadi, 2012 ; Chapellier et al, 2013), il ressort des résultats des entretiens menés dans le cadre de cette étude qu'il existe bien une production et une utilisation des données comptables à des fins de gestion au sein des PME observées. Ce résultat permet d'infirmer l'hypothèse avançant que les pratiques comptables des PME sont rudimentaires, ne servant que pour faire aux obligations fiscales et que les dirigeants des PME n'utilisent pas les données comptables pour piloter leurs entreprises. Autrement dit, le SIC des PME observées est pour partie comptable.

\section{Différenciation des pratiques comptables des PME}

La deuxième question à laquelle ce travail essaie d'apporter des éléments de réponse est relative à l'existence de configurations diverses en matière de SIC des PME marocaines. Les résultats de notre étude montrent que les pratiques comptables des PME observées sont aussi bien différentes 
que diversifiées. Une différence qui concerne les trois dimensions du SIC que sont l'infrastructure comptable, la production et l'utilisation des données comptables. Ce constat confirme les résultats des recherches menées en la matière (Chapellier, 1994; Affes et Chabchoub, 2007; Chapellier et Mohammed, 2010 ; Mohammed, 2010 ; Chapellier et Benhammadi, 2012 ; Chapellier et al, 2013).

\section{Contingences des pratiques comptables des dirigeants de PME}

Ce travail a pour objectif de déterminer dans quelle mesure certaines contingences d'ordre structurel et comportemental sont susceptibles d'expliquer la différenciation des SIC des PME. Les résultats des entretiens menés dans le cadre de cette étude montrent que la contingence structurelle ne suffit pas pour expliquer la variabilité des pratiques comptables au sein des PME. Il semble que les caractéristiques d'ordre comportemental sont de nature à expliquer également cette différenciation.

Il ressort des entretiens menés dans la cadre de cette étude que les caractéristiques structurelles de la PME peuvent expliquer la différenciation des pratiques comptables. Cinq éléments d'ordre structurel sont repérés comme facteurs potentiel pouvant expliquer cette différenciation. Il s'agit de la taille, l'endettement, le statut juridique, le secteur d'activité et l'incertitude perçue de l'environnement. D'autres recherches réalisées antérieurement ont pareillement confirmé l'influence de ces déterminants contextuels sur la différenciation des SIC des PME (Chapellier, 1994 ; Lavigne, 1996, 1999, 2002 ; Lavigne et Saint-pierre, 2002 ; Lassoued et Abdelmoula, 2006 ; Affes et Chabchoub, 2007 ; Ngongang, 2007 et 2013 ; Chapellier et Mohammed, 2010 ; Mohammed, 2010 ; Chapellier et Ben Hammadi, 2012 ; Chapellier et al, 2013).

\section{La taille}

Les résultats de l'étude de Lavigne (2002) ont montré qu'en matière d'états financiers annuels, les PME de grande taille choisissent des conventions comptables autres que les règles fiscales et que les entreprises ayant recours à l'audit sont de taille moyenne supérieure. S'agissant des pratiques de comptabilité de management, les PME qui calculent informatiquement leurs prix de revient et qui préparent des budgets de caisse sont de taille moyenne supérieure. Pour ce qui est de la diffusion interne des résultats financiers, l'étude conclut que les PME de taille moyenne supérieure ne centralisent pas l'information au niveau supérieur et décentralisent l'information jusqu'au niveau inférieur des employés. L'étude de Lavigne et Saint-Pierre (2002) a montré que les entreprises qui ont une taille moyenne supérieure disposent d'un SIC ayant un fort indice d'importance. 
La taille est un facteur explicatif de l'utilisation des données comptables selon l'enquête de Lassoued et Abdelmoula (2006). En effet, le degré d'utilisation des données comptables augmente avec l'augmentation de la taille de l'entreprise. La complexité du SIC des PME est largement influencée par le facteur taille selon Affes et Chabchoub (2007). Ngongang (2007) a examiné les déterminants du SIC des PME. Son étude montre que la taille de l'entreprise a un effet statistiquement significatif sur le SIC.

En essayant de déterminer les facteurs de contingence susceptibles d'expliquer la complexité du SIC des PME industrielles, Chapellier et Mohammed (2010) ont montré que la taille figure en premier lieu comme facteur déterminant de la complexité du SIC au sein de cette catégorie d'entreprises.

Dans le but d'identifier un certain nombre de facteurs susceptibles d'influencer le système de données comptables (SDC) des PME, les résultats de l'étude de Chapellier et Ben Hamdi (2012) ont montré l'existence d'une corrélation positive entre la taille de l'entreprise et la complexité du SDC.

Ngongang (2013) examine l'impact d'un certain nombre de facteurs structurels sur la pratique de comptabilité analytique et le système de contrôle de gestion des PME. La pratique de comptabilité analytique est influencée par la taille de l'entreprise. Le système de contrôle de gestion, représenté par le degré d'importance accordé par le contrôleur de gestion aux budgets et à l'analyse des écarts, est significativement lié à la taille de l'entreprise.

\section{L'endettement}

Les études portant sur l'endettement ont abouti à des résultats mitigés. Une première catégorie d'études confirment l'influence de ce facteur sur le SIC des PME. Une deuxième catégorie, quant à elle, ne montrent aucun lien entre le degré d'endettement et la complexité du SIC.

Lavigne (1996) avance l'hypothèse selon laquelle «Les PME les moins endettées préparent leurs états financiers selon les règles fiscales ». Les résultats de son étude confirment cette hypothèse, au moins pour les entreprises de l'échantillon. Il y a un lien statistiquement significatif entre le ratio d'endettement et la tendance à préparer les états financiers. L'enquête de Lavigne (1999) montre que l'endettement est un des déterminants des pratiques de la comptabilité générale. Le même auteur montre que l'endettement est également un facteur explicatif de la préparation des budgets de caisse par les PME (Lavigne, 2002). Lavigne et Saint-Pierre (2002) ont confirmé ce dernier résultat. En outre ils ont montré que l'indice d'importance du SIC est influencé par le niveau d'endettement de l'entreprise. 
De leur part, Chapellier et Mohammed (2010) ne trouvent aucune relation significative entre la différenciation du SIC et le degré d'endettement de l'entreprise. Chapellier et Ben Hamdi (2012) ne confirment pas l'hypothèse de l'existence d'une corrélation positive entre le degré d'endettement des PME et la complexité de leurs systèmes de données comptables (SDC).

\section{Le secteur d'activité}

Selon Lassoued et Abdelmoula (2006), le secteur d'activité n'explique pas le degré d'utilisation des données comptables. Pour Ngongang (2007), l'informatisation du SIC est dépendante du type d'activité. Ce dernier ne constitue pas un déterminant de la fréquence de production des informations financières. En matière de contrôle de gestion, le secteur d'activité explique la fréquence de production des informations liées aux budgets (Ngongang, 2007). D'après Ngongang (2013), il existe un lien statistiquement significatif entre le secteur d'activité et la pratique de la comptabilité analytique ainsi que le recours du contrôleur de gestion à ce type de comptabilité.

Bien que cette recherche a mis en évidence la dépendance de la différenciation des pratiques comptables des caractéristiques structurelles des PME, il n'en demeure pas moins que les résultats ont abouti également à l'influence éventuelle des caractéristiques comportementales sur l'existence de configurations diverses en matière de SIC des PME. Les entretiens ont relevé l'importance des caractéristiques des acteurs comptables. Il s'agit notamment du dirigeant (âge, niveau et type de formation) et du responsable du service comptable interne (niveau et type de formation). D'autres études portant sur le SIC des PME ont également confirmé cette relation significative entre la différenciation des pratiques comptables des PME et certains facteurs d'ordre comportemental (Lavigne, 2002 ; Lavigne et SaintPierre, 2002 ; Lassoued et Abdelmoula, 2006 ; Affes et Chabchoub, 2007 ; Ngongang, 2007 ; Chapellier et Mohammed, 2010, Mohammed, 2010 ; Chapellier et Ben Hamdi, 2012).

\section{La formation du dirigeant}

Les résultats de l'étude menée par Lavigne (2002) et Lavigne et Saint-Pierre (2002) montrent l'absence de lien entre la formation du dirigeant et les conventions comptables retenues par les PME. Selon les mêmes auteurs, une forte proportion des PME de leurs échantillons dont les dirigeants disposent d'une formation universitaire ne centralise pas l'information comptable au niveau supérieur. Il s'avère également que les conventions comptables retenues par les dirigeants de PME ayant une formation de niveau universitaire ne sont pas alignées sur les dispositions 
fiscales (Lavigne, 2002). Cependant, Lavigne (2002) ne trouve aucune liaison significative entre le niveau de formation du dirigeant et les pratiques relatives à la comptabilité de management. L'enquête de Lavigne et SaintPierre (2002) établit une corrélation positive entre l'indice d'importance du SIC et le niveau de formation du dirigeant.

Pour Lassoued et Abdelmoula (2006), il existe une liaison statistiquement significative entre l'utilisation des données comptables et le niveau de formation du dirigeant. Pour Affes et Chabchoub(2007), les PME dont les dirigeants ont une formation en comptabilité, en finance ou en gestion, disposent de SIC plus complexe que les autres. Par conséquent, le niveau de formation est un facteur explicatif de la différenciation du SIC des PME selon ces deux auteurs.

Dans la même perspective, Le type de formation du dirigeant constitue également un déterminant des pratiques comptables des PME selon Ngongang (2007) qui montre l'existence d'une liaison entre le type de formation du dirigeant (gestionnaire/non-gestionnaire) et le SIC.

Cependant, aucune liaison statistiquement significative n'a pu être établie entre la complexité du SIC et le type de formation du dirigeant (gestionnaire/non-gestionnaire) selon Chapellier et Mohammed (2010). Selon ces deux auteurs, le niveau de formation du dirigeant constitue, par contre, un déterminant de la différenciation du SIC des PME.

L'étude de Chapellier et Ben Hamadi (2012) montre que la complexité du SDC dépend du niveau de formation du dirigeant. En effet, les dirigeants ayant un niveau de formation élevé disposent de SDC plus complexes. Le type de formation explique également la complexité du SDC dans la mesure où le SDC est plus complexe dans les PME dont les dirigeants ont une formation en gestion.

\section{L'âge du dirigeant}

Lassoued et Abdelmoula (2006) testent l'hypothèse selon laquelle l'utilisation des données comptables est moindre chez les dirigeants les plus âgés. Les résultats de leur recherche infirment cette hypothèse puisqu'ils constatent l'absence de relation significative entre l'âge du dirigeant et l'utilisation des données comptables. Ces résultats sont également confirmés par Ngongang (2007). Par contre Affes et Chabchoub(2007) affirment que l'âge du dirigeant explique la complexité du SIC.

\section{Le responsable du service comptable interne}

Les études de Lavigne (2002) et de Lavigne et Saint-Pierre (2002) ne montrent aucune relation entre le responsable de la fonction comptabilité et le type de conventions comptables retenues par les PME. Le niveau de formation du comptable interne est déterminant du type de conventions 
comptables selon les mêmes auteurs. Ces auteurs affirment l'influence du niveau de formation du comptable interne sur la pratique d'audit, le calcul informatique des prix de revient et la décentralisation de l'information à un niveau inférieur des employés. Le niveau de formation du comptable interne est un facteur explicatif de l'importance du SIC selon Lavigne et Saint-Pierre (2002).

Selon Lassoued et Abdelmoula (2006), il existe une corrélation positive entre le niveau de formation du comptable salarié et l'utilisation des données comptables. Selon ces auteurs, l'utilisation des données comptables est également dépendante de la mission du comptable salarié. Ce résultat est confirmé par Chapellier et Mohammed (2010) qui trouvent une relation statistiquement significative entre la mission du comptable interne et la différentiation du SIC. L'effet du degré d'implication de l'expert-comptable sur la complexité du SIC est confirmé selon Chapellier et Mohammed (2010).

\section{Conclusion}

Ce travail avait pour objectif d'essayer d'apporter des éléments de réponse à la question de la pertinence des données comptables pour les dirigeants des PME marocaines. Pour cela une étude exploratoire du SIC de PME a été réalisée à travers des entretiens individuels semi-directifs menés auprès des témoins privilégiés (experts-comptables) et du public directement concerné (dirigeant et comptables internes) afin de décrire les différentes pratiques comptables des PME d'une part, et de faire ressortir des déterminants potentiels qui peuvent expliquer la différenciation de ces pratiques, d'autre part.

Les résultats des entretiens menés ont permis d'aboutir aux conclusions suivantes :

- le système d'information des dirigeants de PME marocaines est pour partie comptable ;

- les pratiques comptables des PME marocaines sont différentes et diversifiées ;

- deux types de facteurs pourraient expliquer cette différenciation :

- des facteurs d'ordre structurel tels que la taille, le secteur d'activité, le statut juridique, l'endettement et l'environnement ;

- des facteurs d'ordre comportemental tels que le profil du dirigeant (Age, niveau et type de formation) et le profil du comptable interne (niveau et type de formation).

L'approche méthodologique retenue axée sur les entretiens semidirectifs ne permet pas, certes, d'affirmer l'influence de ces facteurs sur la différenciation du SIC des PME marocaines. Toutefois, elle constitue une base pour définir les aspects à prendre en considération pour établir des 
hypothèses qui seront confirmées ou infirmées par la suite dans le cadre d'une prochaine enquête quantitative auprès d'une population élargie de PME.

\section{References:}

1. Affes, H. et Chabchoub, A. (2007). Le système d'information comptable : les déterminants de ses caractéristiques et son impact sur la performance financière des PME en Tunisie. La Revue des sciences de gestion, Direction et gestion, nos 224-225, 59-67.

2. Bergeron, H. Boulerne, S. Roy, C. et Wolff, D. (2010). Identification des enjeux prioritaires des PME dans le but d'établir un tableau de bord pour leur gestion du développement durable. $31^{\text {ème }}$ Congrès annuel de l'Association Francophone de Comptabilité, Nice.

3. Campenhoudt, L.V. et Quivy, R. (2011). Manuel de recherche en sciences sociales (4 ${ }^{\mathrm{ème}}$ édition). Dunod.

4. Chapellier, P. (1994). Comptabilités et système d'information du dirigeant de PME : essai d'observation et d'interprétation des pratiques (Thèse de doctorat de sciences de gestion). Université de Montpellier II.

5. Chapellier, P. et De Montgolfier, C. (1995). Une synthèse des approches méthodologiques du système d'information comptable. $6^{\text {ème }}$ Congrès annuel de l'Association Francophone de Comptabilité, Montpellier.

6. Chapellier, P. (1997). Profils de dirigeants et données comptables de gestion en PME. Revue internationale P.M.E. : économie et gestion de la petite et moyenne entreprise, vol. 10, $\mathrm{n}^{\circ} 1,9-41$.

7. Chapellier, P. et Mohammed, A. (2010). Les pratiques comptables des PME syriennes dans un contexte de libération de l'économie. $31^{\mathrm{ème}}$ Congrès de l'Association Francophone de comptabilité, Nice.

8. Chapellier, P. et Ben Hamadi, Z. (2012). Le système de données comptables des dirigeants de PME tunisiennes : complexité et déterminants. Management internationa, vol. 16, $\mathrm{n}^{\circ}$ 4, 151-167.

9. Chapellier, P. Mohammed A. et Teller, R. (2013). Le système d'information comptable des dirigeants de PME syriennes: complexité et contingences. Management et Avenir, $\mathrm{N}^{\circ} 65,48-72$

10. Chouigui, H. (2007). Les PME exportatrices face au défi chinois: cas multiple des PME manufacturières québécoises (Mémoire de Maitrise en administration des affaires). Université du Québec à Trois Rivières.

11. D'Amboise, G. et Bakanibona, A. (1990). La planification dans les PME : une synthèse de résultats empiriques : conclusions et 
recommandations. Revue internationale P.M.E. : économie et gestion de la petite et moyenne entreprise, vol. $3, \mathrm{n}^{\circ} 2,147-166$.

12. Drain, M.C. (2014). Désalignement des usages $d u$ système d'information dans la création et la propagation des difficultés au sein des PME : cas du secteur agro-alimentaire (Doctorat en sciences de gestion). Conservatoire National des Arts et Métiers - CNAM.

13. Ghorbel, J. (2013). Le design du système d'information comptable dans les PMI tunisiennes : une modélisation contingente. $11^{\text {ème }}$ Congrès International Francophone en Entrepreneuriat et PME, Brest.

14. Gravard-Perret, M.L. Gotteland, D. Haon, C. et JOLIBERT, A. (2012). Méthodologie de la recherche en sciences de gestion $\left(2^{\text {ème }}\right.$ édition). Pearson.

15. Julien, P.A. (1990). Vers une typologie multicritère des PME. Revue internationale P.M.E. : économie et gestion de la petite et moyenne entreprise, vol. 3, $\mathrm{n}^{\circ} 3-4,411-425$.

16. Julien, P.A. (2008). Trente ans de théorie en PME : de l'approche économique à la complexité. Revue internationale P.M.E. : économie et gestion de la petite et moyenne entreprise, vol. 21, $\mathrm{n}^{\circ}$ 2, 119-144.

17. Lassoued, K. et INES, A. (2006). Les déterminants des systèmes d'information comptables dans les PME : une recherche empirique dans le contexte tunisien. $27^{\text {ème }}$ congrès de l'AFC, Tunis.

18. Lavigne, B. (1996). Les états financiers des PME sont-ils seulement utiles pour des fins fiscales ? $64^{\text {ème }}$ Congrès de l'ACFAS. Université McGill.

19. Lavigne, B. (2002). Contribution à l'étude de la genèse des systèmes d'information comptable des PME : une approche empirique. Actes du $23^{\text {ème }}$ Congrès de l'Association Francophone de comptabilité, Toulouse.

20. Lavigne, B. et St-Pierre, V. (2002). Association entre le système d'information comptable des PME et leur performance financière.

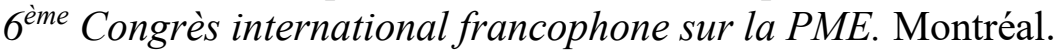

21. Marchesnay, M. (1988). La mercatique de la petite entreprise. Revue internationale P.M.E. : économie et gestion de la petite et moyenne entreprise, vol. 1, $\mathrm{n}^{\circ}$ 3-4, 259-276.

22. Marchesnay, M. (1991). La P.M.E. : une gestion spécifique. Economie rurale, Volume 206, $\mathrm{n}^{\circ} 1,11-17$.

23. Mohammed, A. (2010). Etude du Système d'Information Comptable des PME syriennes. Observation et essai de compréhension des pratiques (Doctorat en sciences de gestion). Université de Nice Sophia Antipolis. 
24. Ngongang, D. (2007). Analyse des facteurs déterminants du système d'information comptable et des pratiques comptables des PME Tchadiennes. La Revue des Sciences de Gestion, n²24-225, 49-57.

25. Ngongang, D. (2013). Système d'information comptable et contrôle de gestion dans les entreprises camerounaises. La Revue Gestion et Organisation, $\mathrm{n}^{\circ}$ 5, 113-120.

26. OCDE. (2004). Faciliter l'accès des PME aux marchés internationaux. $2^{\text {ème }}$ conférence de l'OCDE des ministres en charge des PME. Istanbul.

27. Torres, O. (1998). Vingt-cinq ans de recherche en gestion de la PME : une discipline entre courants et contre-courants. Dans O. Torres (sous la coordination), PME : De nouvelles approches: Editions Economica.

28. Zian, H. (2013). Contribution à l'étude des tableaux de bord dans l'aide à la décision des PME en quête de performances (Doctorat ès sciences de gestion). Université Montesquieu - Bordeaux IV. 